\title{
Impact of Strain on the Superconducting Properties of Strongly Underdoped $\mathrm{La}_{2-x} \mathrm{Sr}_{x} \mathrm{CuO}_{4}$ Thin Films
}

\author{
I. Zaytseva, M.Z. Cieplak, W. Paszkowicz, A. Abal'oshev and M. Berkowski \\ Institute of Physics, Polish Academy of Sciences, Al. Lotników 32/46, 02-668 Warsaw, Poland
}

\begin{abstract}
Using pulsed laser deposition we have grown films of $\mathrm{La}_{2-x} \mathrm{Sr}_{x} \mathrm{CuO}_{4}$ with $x$ in close vicinity of the superconductor-insulator transition, $x=0.051$ and $x=0.048$, on $\mathrm{SrLaAlO}_{4}$ substrates, and of different thickness $d$ (from $25 \mathrm{~nm}$ to $250 \mathrm{~nm}$ ). The X-ray diffraction shows that for each $d$ the films grow with variable degree of compressive in-plane strain, with the largest strain achieved in thinnest films. The resistivity measurements show strong enhancement of superconductivity with increasing strain, so that the onset of superconductivity at temperature as high as $27 \mathrm{~K}$ is observed. With increasing strain the character of resistivity changes from the insulating to metallic.
\end{abstract}

PACS: 74.25.F-, 74.62.En, 74.78.-w

\section{Introduction}

The effect of biaxial pressure on the superconducting transition temperature $\left(T_{c}\right)$ is frequently used as a probe of the superconducting mechanism. In high temperature superconductors it is usually observed that the $T_{c}$ is enhanced by the uniaxial pressure applied parallel to the $a$ crystallographic axis (in the $\mathrm{CuO}_{2}$ plane), while it is suppressed by the pressure applied parallel to the $c$-axis [1]. The method particularly useful for the evaluation of these effects is the study of thin superconducting films subject to epitaxial strain induced by the lattice mismatch with the substrate. One of the best known examples is the case of $\mathrm{La}_{2-x} \mathrm{Sr}_{x} \mathrm{CuO}_{4}$ (LSCO) films grown on $\mathrm{SrLaAlO}_{4}$ (SLAO) substrate, in which compressive inplane strain is induced [2-7]. Substantial enhancement of the $T_{c}$ has been reported for optimally doped, slightly underdoped, and undoped $(x=0)$ films deposited on SLAO substrates and cooled in $\mathrm{O}_{3}$ atmosphere $[3,7]$. While originally this enhancement has been attributed entirely to substrate-induced strain [3], a later suggestion has been made that the excess oxygen intercalation is responsible [7]. Our study of thickness-dependent strain in optimally-doped LSCO suggests that both the strain, and the oxygenation of films play important role [6]. Finally, it has been suggested that the strong sensitivity of the $T_{c}$ to strain may be traced to the strain-induced modifications of the local lattice distortions [8], which probably modify, in turn, the distribution of nanoscale charge inhomogeneities naturally existing in LSCO.

The strain-induced effects should be even more pronounced in strongly underdoped LSCO with $x \approx 0.05$, in close vicinity of the superconductor-insulator transition (SIT), since in this case the concentration of carriers is small, so that the charge inhomogeneities may become more significant. Therefore, one may expect that the SIT could be shifted by the strain. Study of this effect may provide the insight into the nature of the nucleation of superconductivity at the SIT. Motivated by this idea, in the present work we study the effect of epitaxial strain on the superconductivity in strongly underdoped LSCO films, grown on the SLAO substrates. For the growth we use two ceramic targets of LSCO with $x$ in close vicinity of the SIT, one superconducting $(x=0.051)$, and the other non-superconducting $(x=0.048)$.

\section{Experimental details}

Epitaxial LSCO thin films are deposited from stoichiometric ceramic targets by pulsed laser deposition using $\mathrm{Nd}$ :YAG laser with 4 th harmonic generation $(\lambda=$ $266 \mathrm{~nm})$, repetition rate $1 \mathrm{~Hz}$ and energy density $1.5 \mathrm{~J} / \mathrm{cm}^{2}$ at the target surface. During deposition the SLAO substrates are held at temperature $760^{\circ} \mathrm{C}$ in the oxygen atmosphere of 300 mTorr. After deposition, the $\mathrm{O}_{2}$ pressure in the chamber is increased to 500 Torr, and the films are slowly cooled down to room temperature with a rate of $3 \mathrm{~K}$ per minute. The structural quality of the films has been studied by X-ray diffraction using high-resolution Philips XPert MRD Alpha 1 Pro diffractometer. From the data the $c$-axis lattice parameter is extracted. The superconducting transition temperature has been measured using a standard four-probe method by DC measurements in the temperature range 5 to $300 \mathrm{~K}$.

The $a$-axis (in-plane) lattice parameter of SLAO substrates is equal to $3.754 \AA$. This value is about $0.79 \%$ smaller than the in-plane lattice parameter of LSCO with $x=0.048$, which equals to $3.784 \AA$, and about $0.82 \%$ smaller than the in-plane lattice parameter of LSCO with $x=0.051$, which equals to $3.785 \AA$. Therefore, all films grown on SLAO are subject to compressive in-plane strain. It has been shown by our previous studies $[5$, $6]$ that this results in reduction of $a$ lattice parameter, and simultaneous increasing of $c$ lattice parameter. Since these changes are strictly correlated, in order to quantify the strain induced by the lattice mismatch we may use 
the parameter $\varepsilon=c_{f} / c_{b}-1$, where $c_{f}$ and $c_{b}$ are $c$ axis parameters in the film and in the bulk, respectively. Therefore, the positive $\varepsilon$ indicates expansion along the $c$ axis and in-plane compression, while negative $\varepsilon$ indicates $c$-axis compression and in-plane expansion.

For comparison, we also grow the films on substrates which should induce tensile in-plane strain. The substrates are SAT/CAT/LA, with the chemical formula $\left(\mathrm{SrAl}_{0.5} \mathrm{Ta}_{0.5} \mathrm{O}_{3}\right)_{7}\left(\mathrm{CaAl}_{0.5} \mathrm{Ta}_{0.5} \mathrm{O}_{3}\right)_{0.1}\left(\mathrm{LaAlO}_{3}\right)_{0.2}$, and in-plane lattice parameter $a$ equal to $3.876 \AA$, i.e. about $2 \%$ larger than $a$ in LSCO for both values of $x$.

\section{Results and discussion}

Figure 1 shows the dependence of strain $\varepsilon$ on the film thickness $d$ for films deposited on two types of substrates, SLAO and SAT/CAT/LA. The data above $\varepsilon=0$ line are for films deposited on SLAO, while the data below this line are for films on SAT/CAT/LA. This confirms our expectation that the films grown on SLAO or $\mathrm{SAT} / \mathrm{CAT} / \mathrm{LA}$ exhibit compressive and tensile in-plane strain, respectively.

However, in case of SLAO substrate for each group of films of any given $d$ we obtain films with $\varepsilon$ ranging from small to large. The maximal value of $\varepsilon$ which may be achieved for each $d$ increases with decreasing film thickness as indicated approximately by dotted line drawn on the figure. We conclude that the largest compressive inplane strain may be reached in thinnest films, consistent with the expectation for the substrate-induced strain. On the other hand, for each $d$ we also observe films with smaller strain. The reduction of strain is most likely the results of the oxygen deficiency in the films. The oxygen deficiency in the LSCO leads to the decrease in $c$ and increase in $a$ lattice parameter, what is in direct competition with the substrate-induced compressive strain [9]. The results described here are similar to the results observed by us previously for LSCO with $x=0.15$ [6].

It is interesting to note that the films grown on $\mathrm{SAT} / \mathrm{CAT} / \mathrm{LA}$ show less strain variability. This is presumably because in this case the oxygen deficiency and the substrate-induced strain both lead to c-axis decrease in the films.

We now turn attention to the superconducting properties. We define $T_{c}^{o n}$ as the onset of superconductivity, i.e. the temperature at which the resistance starts to drop below the normal-state resistance value. We note first that the ceramic target with $x=0.048$ is nonsuperconducting, while the one with $x=0.051$ shows $T_{c}^{o n}$ less than $4 \mathrm{~K}$. Figure 2 shows the dependence of $T_{c}^{o n}$ on $\varepsilon$ for a series of films with various $d$ for two $x$ values. We observe $T_{c}^{o n}$ for the films in which $\varepsilon$ is positive and larger than 0.1 . On the other hand, no trace of superconductivity is present in the films with tensile strain. Moreover, the data in Fig. 2 are quite scattered, nevertheless on average $T_{c}^{o n}$ increases with the increase of $\varepsilon$ for both $x$ values. The most remarkable is the appearance of $T_{c}^{o n}$ with a value as high as $27 \mathrm{~K}$ in the film with $x=0.048$

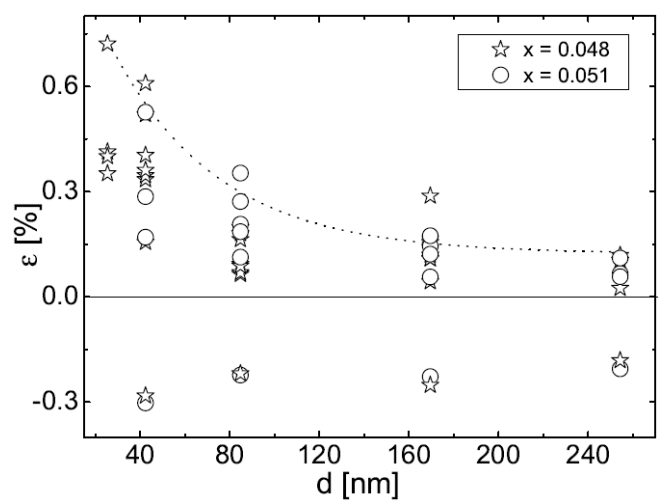

Fig. 1. The dependence of strain $\varepsilon$ on the films thickness. The data with $\varepsilon>0(\varepsilon<0)$ are for films grown on SLAO (SAT/CAT/LA) substrates.

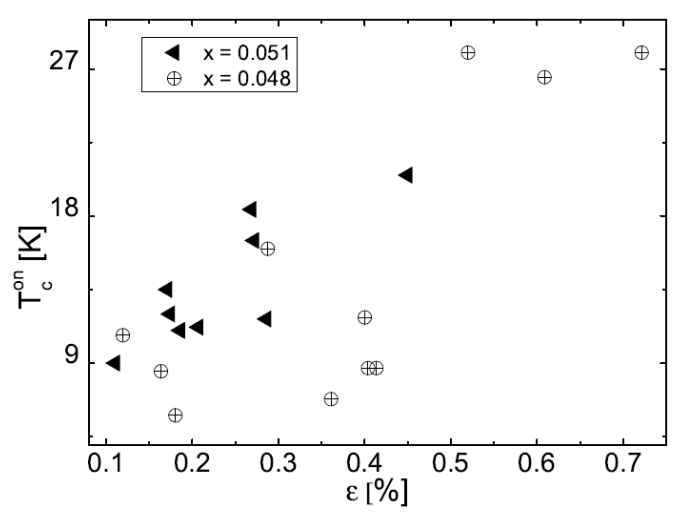

Fig. 2. The dependence of $T_{c}^{o n}$ on the strain $\varepsilon$.

and $\varepsilon=0.72$. This data indicate that compressive strain strongly enhances superconductivity.

Figure 3 shows the behavior of the resistance normalized to the room temperature resistance, $R / R_{292 \mathrm{~K}}$, in a wide $T$-range for films with $x=0.048$. We see that with increasing $\varepsilon$ the resistance evolves from insulating-like to metallic-like and superconducting. Just like in case of the dependence of $T_{c}^{o n}$ on $\varepsilon$, there is some scatter in the magnitude of $R / R_{292 \mathrm{~K}}$ between films, but the general trend is that with increasing $\varepsilon R / R_{292 \mathrm{~K}}$ decreases. However, the transition to the superconducting state is not complete in these films. This is shown in more detail in the inset to Fig. 3 for films with the largest $\varepsilon$. Using $I-V$ measurement at low $T$ we have confirmed that these samples do not become fully superconducting. A possible explanation is that the superconducting state remains confined to isolated islands, which are immersed in insulating or metallic matrix, similar to granular superconductivity. The scatter of the data is caused by the randomness of such system.

In case of films with $x=0.051$ we observe two different behaviors. One group of films (Fig. 4a) shows the behavior similar to films with $x=0.048$, i.e. with increasing $\varepsilon R / R_{292 \mathrm{~K}}$ decreases and becomes metallic-like, 


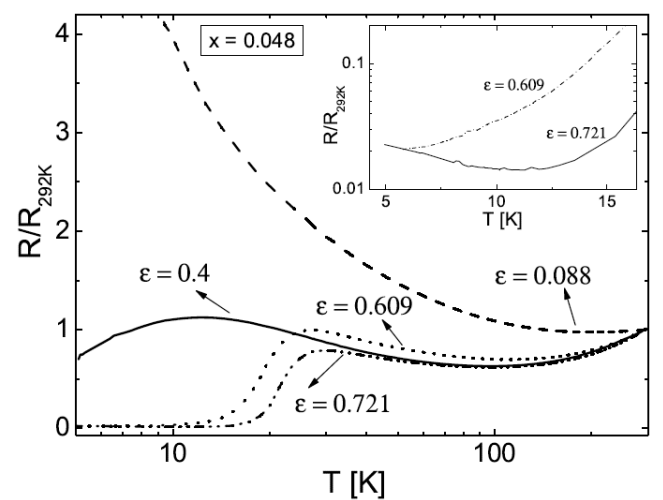

Fig. 3. Normalized resistance versus $T$ on logarithmic scale for films with different $\varepsilon$ and $x=0.048$. Inset: normalized resistance on a logarithmic scale vs. $T$ for $\varepsilon=0.609$ and $\varepsilon=0.721$.

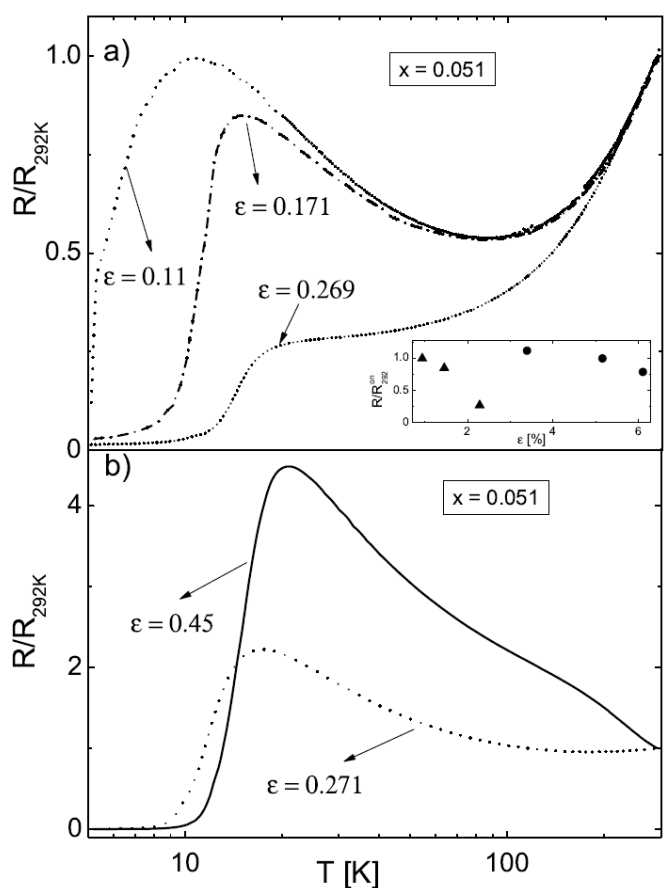

Fig. 4. Normalized resistance versus $T$ on logarithmic scale for films with different $\varepsilon$ and $x=0.051$, for samples with metallic character of the resistance (a), and samples with semiconducting character of the resistance (b). Inset shows $R / R_{292 \mathrm{~K}}$ at the onset of superconducting transition vs. $\varepsilon$.

but the transition to superconducting state is not complete. Note that the magnitude of $R / R_{292 \mathrm{~K}}$ in these films is comparable to those with $x=0.048$ but it is achieved at lower values of $\varepsilon$ (inset Fig. 4a). This suggests that the increased concentration of carriers contributes to better electrical conduction in the normal state. However, the nucleation of superconductivity still seems to be confined to more or less isolated islands.

Interestingly, we also observe another group of films (Fig. 4b), for which $R / R_{292 \mathrm{~K}}$ is much higher, but these films become completely superconducting at low $T$. In this case the correlation between the decrease of $R / R_{292 \mathrm{~K}}$ and increase of $\varepsilon$ is lost, although the value of $T_{c}^{o n}$ still increases with increasing $\varepsilon$. This seems to indicate that the strain is not the only factor which determines the magnitude of the resistance in some of these ultrathin films. Some other factors which may contribute include oxygen vacancies or other defects, which may strongly affect the nature of the metallic or insulating matrix, or the size of the superconducting areas. Further studies are needed to evaluate in more detail the origins of additional factors which affect superconductivity.

In conclusion, we have investigated the effect of strain on superconductivity in thin films of LSCO with $x$ in close vicinity of the SIT. We find that the compressive strain introduced by the lattice mismatch with SLAO substrates strongly enhances superconductivity, leading to appearance of strain-induced onset of superconductivity at temperatures as high as $27 \mathrm{~K}$. This is accompanied by the evolution of the normal-state resistance from insulating to metallic.

\section{Acknowledgments}

This work was partially supported by Polish National Science Center grant 2011/01/B/ST3/00462.

\section{References}

[1] J.-S. Zhou, J.B. Goodenough, H. Sato, M. Naito, Phys. Rev. B 59, 3827 (1999).

[2] M.Z. Cieplak, M. Berkowski, S. Guha, E. Cheng, A.S. Vagelos, D.J. Rabinowitz, B. Wu, I.E. Trofimov, P. Lindenfeld, Appl. Phys. Lett. 65, 3383 (1994).

[3] J.P. Locquet, J. Perret, J. Fompeyerine, E. Machler, J.W. Seo, G. Van Tendeloo, Nature (London) 394, 453 (1998).

[4] H. Sato, M. Naito, Physica C 274, 221 (1997); H. Sato, A. Tsukada, M. Naito, A. Matsuda, Phys. Rev. B 61, 12447 (2000); H. Sato, A. Tsukada, M. Naito, A. Matsuda, Phys. Rev. B 62, R799 (2000).

[5] M.Z. Cieplak, M. Berkowski, A. Abal'oshev, S. Guha, Q. Wu, Supercond. Sci. Technol. 19, 564 (2006).

[6] I. Zaytseva, M.Z. Cieplak, A. Abal'oshev, M. Berkowski, V. Domukhovski, W. Paszkowicz, A. Shalimov, Acta Phys. Pol. A 111, 185 (2007).

[7] I. Bozovic, G. Logvenov, I. Belca, B. Narimbetov, I. Sveklo, Phys. Rev. Lett. 89, 107001 (2002).

[8] H. Oyanagi, A. Tsukada, M. Naito, N.L. Saini, Phys. Rev. B 75, 024511 (2007).

[9] W. Si, H.-C. Li, X.X. Xi, Appl. Phys. Lett. 74, 2839 (1999). 